\title{
Prophylactic antenatal corticosteroid before elective cesarean delivery at or near term: a retrospective study
}

\author{
Sana Sharfuddin 1 , Anup Pradhan ${ }^{1 *}$, Md Nazar Imam²
}

\begin{abstract}
${ }^{1}$ Department of Obstetrics and Gynecology, ${ }^{2}$ Department of Surgery, Sikkim Manipal Institute of Medical Sciences, Gangtok, Sikkim, India
\end{abstract}

Received: 09 January 2019

Accepted: 05 February 2019

*Correspondence:

Dr. Anup Pradhan,

E-mail: anuppradhan@ live.com

Copyright: () the author(s), publisher and licensee Medip Academy. This is an open-access article distributed under the terms of the Creative Commons Attribution Non-Commercial License, which permits unrestricted non-commercial use, distribution, and reproduction in any medium, provided the original work is properly cited.

\begin{abstract}
Background: While the role of antenatal steroids administration to mother is proved in reducing neonatal morbidity and mortality in preterm gestation secondary to respiratory distress and hyaline membrane disease its role in patients undergoing elective cesarean deliveries at or near term appears to be controversial.

Methods: This was a retrospective observational study in which women who have undergone elective cesarean delivery between 34-37 weeks of gestation were included. Those women who received two intramuscular injections of $12 \mathrm{mg}$ betamethasone $24 \mathrm{~h}$ apart were included in group S whereas the women who didn't receive such injections were included in group B. Outcome measures were incidence of transient tachypnea of the newborn, hyaline membrane disease and NICU admissions due to respiratory distress.

Results: Neonates in the treatment group had a statistically significant lower overall incidence of transient tachypnea of newborn, respiratory distress syndrome and NICU admissions. The incidence of transient tachypnea of newborn and respiratory distress in Group $\mathrm{S}$ was $5 \%$ and $8.33 \%$ respectively where as TTN and respiratory distress was seen in $18.33 \%$ and $28.33 \%$ neonates in the group N. The over NICU admission rates, due to respiratory distress in group S and Group $\mathrm{N}$ were found to be $8.33 \%$ and $28.33 \%$ respectively.

Conclusions: Antenatal administration of corticosteroids to women at or near term (34-37 weeks) is found to have a beneficial effect in reducing neonatal morbidity and NICU admissions secondary to respiratory distress in women undergoing elective CS.
\end{abstract}

Keywords: Antenatal corticosteroids, Elective cesarean section, Respiratory distress, Transient tachypnea of newborn

\section{INTRODUCTION}

Cesarean deliveries are associated with increased incidence of respiratory distress in new born babies. In majority of the neonates this respiratory distress is due to transient tachypnoea of new born which usually mild and self-limiting cause of respiratory distress is and is likely to settle on its own. ${ }^{1}$ But in some other neonates there can be frank respiratory distress secondary to atelectasis due to surfactant deficiency. ${ }^{2}$ Neonates developing respiratory distress immediately after birth due to surfactant deficiency may require NICU admissions and mechanical ventilation and if these neonates are not managed properly there can be increased morbidity in the form of persistent pulmonary hypertension and long term oxygen dependency. Failure to intervene at appropriate time may even prove fatal. ${ }^{3}$

The chances of infants developing respiratory distress are more after cesarean delivery as compared to the infants born through normal vaginal delivery. These neonates are at risk of developing respiratory distress on account of 
surfactant deficiency leading to poor expansion of lungs following birth. ${ }^{4}$ Moreover, there are studies which have found that amongst all the infants born through cesarean deliveries the incidence of respiratory morbidity and mortality is more in infants born through elective cesarean delivery as compared to neonates born following caesarean section after onset of labor. ${ }^{5}$ Prophylactic administration of corticosteroids in preterm as well as near-term pregnancies are responsible for accelerated lung maturity thereby reducing incidence of respiratory distress, admissions in neonatal intensive care units and need for exogenous surfactant treatment6. Various studies have found that the risk of respiratory morbidity decreases significantly at and after 39 weeks so it has been recommended that the elective cesarean delivery if possible, should be deferred till 39 weeks of gestation. ${ }^{7}$ However, this may not be possible in all the cases and elective cesarean section may have to be undertaken in view of various situations. In cases where the risk of continuing pregnancy outweighs the benefits of continuing pregnancy it becomes prudent to deliver the baby to prevent adverse pregnancy outcome. ${ }^{8}$

Role of prophylactic steroid injection is proved in pregnant women before 34 weeks of gestation and who are at the risk of developing preterm labour. There is evidence of reduction in neonatal morbidity due to respiratory distress, intracranial haemorrhage, necrotizing entero-colitis and need for admission to neonatal intensive care unit in these neonates. ${ }^{9}$

The evidence for administration of corticosteroids after 34 weeks is still controversial since there is a lack of properly conducted trials of antenatal corticosteroids in mothers delivered by elective cesarean delivery at this gestational age. Moreover, the adverse effects of antenatal steroid administration such as delayed myelination, long term effects on the setting of the hypothalamo-pituitary axis, reduced birth weight, altered glucose homeostasis and increased incidence of behavioural disorder in early childhood makes it essential that antenatal corticosteroids must be judiciously used taking into account risk and benefits of its administration. ${ }^{10}$

This retrospective observational case control study was conducted to find out whether prophylactic steroid administration before elective cesarean delivery at or near term (between 34-37 weeks of gestation) reduces the incidence of neonatal morbidity associated with respiratory distress and rate of admissions in neonatal intensive care unit for respiratory distress.

\section{METHODS}

This was a retrospective observational case control study of pregnant women with gestational age between $34-37$ weeks of gestation who had undergone elective cesarean delivery. The women who received two intramuscular injections of $12 \mathrm{mg}$ betamethasone 24 hour apart were included in group $\mathrm{S}$ while those women who have not received any dose of steroid were included in group $\mathrm{N}$. Total 120 women with singleton pregnancy were included in this study (60 in each group) on the basis of a predefined inclusion and exclusion criteria.

A Detailed history of present pregnancy with respect to date of last menstrual period, and relevant past history was noted from case records of the patients. If the patient was referred from other hospital, then the indication for such referral was also noted down from case papers. Detailed past history, obstetric and menstrual history, family and personal history were noted down from medical records. Nutritional status, built, height and weight of the patients were also noted in the proforma. Relevant systemic examination was done. Findings of Per abdominal examination was noted down with respect to the height of uterus, position, lie, presence or absence of contractions, presence of FHS and its rate were noted from medical records. Findings of per vaginal examination were noted in all cases. Basic investigations $\mathrm{Hb} \%$, bleeding time, clotting time, blood grouping and Rh typing, HIV, VDRL and HbSAg were recorded. Indication for elective cesarean delivery was also noted. Any maternal risk factors such as maternal diabetes, history of infection during antenatal period, eclampsia or premature rupture of membranes etc were also noted.

All deliveries were attended by pediatrician on call. APGAR score at 1 minute and 5 minute was noted from pediatrician's notes. Need for immediate resuscitation measures and indication for admission to neonatal intensive care unit was ascertained from attending pediatrician's notes. Primary outcome was assessed by need of admission to neonatal intensive care unit for respiratory distress including that due to transient tachypnea of the neonate (TTN) and respiratory distress syndrome (RDS) or need for surfactant therapy and mechanical ventilation.

Patients were discharged as per institutional protocol or on patients request if there were no contraindications to such a discharge. Microsoft office was used for preparation of charts and graphs and Statistical analysis was done using SSPE 22. $\mathrm{P}$ value less than 0.05 was taken as statistically significant.

\section{Inclusion criteria}

- All patients who had undergone elective cesarean delivery between 34-37 weeks of gestation.

- $\quad$ Singleton Pregnancy.

\section{Exclusion criteria}

- Multiple pregnancies

- Gestational age less than 34 weeks.

- Those patients in whom neonatal outcome couldn't be determined from case paper. 


\section{RESULTS}

This was a hospital based retrospective observational case control study conducted in the department of obstetrics and gynecology of a tertiary care medical college. 120 pregnant women who had undergone elective cesarean delivery were included in this study on the basis of a predefined inclusion and exclusion criteria after carefully going through the medical records of the mother and the baby. The women who received two intramuscular injections of $12 \mathrm{mg}$ betamethasone $24 \mathrm{~h}$ apart were included in group $\mathrm{S}$ while those women who have not received any dose of steroid were included in group $\mathrm{N}$. The analysis of the age groups of the patients showed that the mean age of the patients in group $\mathrm{S}$ was $21.23 \pm 3.62$ while the mean age in Group $\mathrm{N}$ was 22.27 \pm 4.12 . There was no statistically significant difference in the age groups of the studied groups $(\mathrm{p}=0.14)$ (Table 1$)$.

Table 1: Age groups of patients in both the groups.

\begin{tabular}{|l|l|l|l|l|}
\hline & \multicolumn{2}{|c}{ Group S } & \multicolumn{2}{c|}{ Group N } \\
\hline 20 years or below & 26 & 43.33 & 23 & 38.33 \\
\hline 21-30 years & 22 & 36.67 & 21 & 35.00 \\
\hline Above 30 years & 12 & 20.00 & 16 & 26.67 \\
\hline Total & 60 & 100 & 60 & 100 \\
\hline
\end{tabular}

The analysis of gestational age of both the groups showed that the mean gestational age of the patients in group $S$ was $35.82 \pm 1.12$ weeks whereas the mean gestational age of the patients in group $\mathrm{N}$ was $34.98 \pm 0.98$ weeks. There was no statistically significant difference in the gestational age of patients in both the groups $(\mathrm{P}=0.46)$ (Table 2).

Table 2: Mean gestational age of the studied cases.

\begin{tabular}{|l|l|l|l|}
\hline & $\begin{array}{l}\text { Mean } \\
\text { gestational } \\
\text { age (weeks) }\end{array}$ & $\begin{array}{l}\text { Std. } \\
\text { deviation }\end{array}$ & Significance \\
\hline Group S & 35.82 & 1.12 & $\mathrm{P}=0.46$ \\
\hline Group N & 34.90 & 1.40 & Not significant \\
\hline
\end{tabular}

Table 3: Gravida of the studied cases.

\begin{tabular}{|c|c|c|c|c|}
\hline \multirow{2}{*}{ Gravida } & \multicolumn{2}{|l|}{ Group S } & \multicolumn{2}{|l|}{ Group N } \\
\hline & No. of cases & $\%$ & No. of cases & $\%$ \\
\hline $1^{\text {st }}$ & 28 & 46.67 & 24 & 40 \\
\hline $2^{\text {nd }}$ & 16 & 26.67 & 18 & 30 \\
\hline $3^{\text {rd }}$ & 14 & 23.33 & 15 & 25 \\
\hline $4^{\text {th }}$ & 2 & 3.33 & 3 & 5 \\
\hline Total & 60 & 100 & 60 & 100 \\
\hline
\end{tabular}

In both the groups majority of the patients were primigravida ( $46.67 \%$ in group $\mathrm{S}$ and $40 \%$ in Group $\mathrm{N}$ ). $16(26.67 \%)$ and $18(30 \%)$ patients were 2nd gravida in Group $\mathrm{S}$ and Group $\mathrm{N}$ respectively (Table 3). The analysis of the indications for elective LSCS showed that majority of elective LSCS in both the groups were done for scar tenderness $(33.33 \%)$. The other common indications for LSCS in patients was found to be cephalopelvic disproportion $(22.50 \%)$, malpresentation (21.67\%) oligohydramnios $(12.50 \%)$, abnormalities on Doppler indicating compromised utero-placental flow (10\%) (Figure 1).

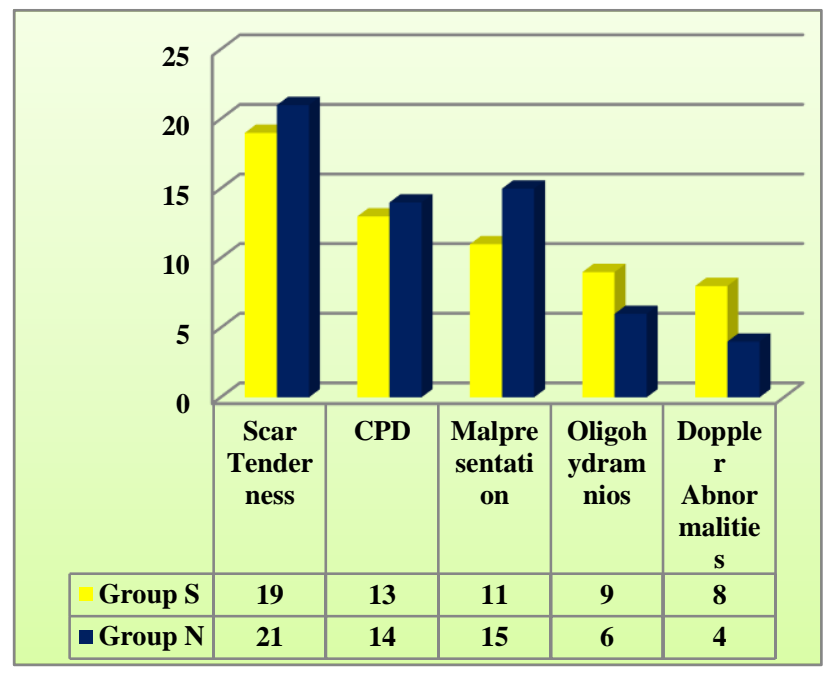

Figure 1: Indications of Elective LSCS in studied cases.

The analysis of birth weights of both the groups showed that the mean birth weight of the newborn babies was $2.61 \pm 0.102 \mathrm{~kg}$ in group $\mathrm{S}$ whereas the mean birth weight of the neonates in group $\mathrm{N}$ was $2.58+/-0.09$. Birth weights of the neonates were comparable and there was no statistically significant difference in between the birth weights of the babies $(\mathrm{P}=0.103)$ (Table 4$)$.

Table 4: Mean birth weights of neonates in studied cases.

\begin{tabular}{|l|l|l|l|}
\hline & $\begin{array}{l}\text { Mean birth } \\
\text { weight (kg) }\end{array}$ & $\begin{array}{l}\text { Std. } \\
\text { deviation }\end{array}$ & Significance \\
\hline Group S & 2.612 & 0.102 & $\mathrm{P}=0.103$ \\
\hline Group N & 2.582 & 0.098 & Not significant \\
\hline
\end{tabular}

The analysis of the neonates for development of transient tachypnoea of newborn showed that in group $\mathrm{N}$ out of 60 neonates $11(18.33 \%)$ neonates developed TTN where as in Group S only $3(5 \%)$ neonates developed TTN. The statistical analysis showed that the incidence of TTN was significantly higher in group of women who didn't receive antenatal steroids $(\mathrm{P}=0.04)$ (Table 5).

Table 5: Incidence of transient tachypnoea of newborn (TTN) in new-borns.

\begin{tabular}{|c|c|c|c|}
\hline & $\begin{array}{l}\text { Neonates with } \\
\text { TTN }\end{array}$ & $\%$ & Significance \\
\hline Group N & 11 & 18.33 & $\mathrm{P}=0.04$ \\
\hline Group S & 3 & $5 \%$ & Significant \\
\hline
\end{tabular}


In group $\mathrm{N} 17(28.33 \%)$ neonates developed respiratory distress where as in Group S only $5(8.33 \%)$ neonates developed respiratory distress. The statistical analysis showed that the incidence of respiratory distress was significantly higher in group of women who didn't receive antenatal steroids $(\mathrm{P}=0.008)$ (Table 6).

Table 6: Incidence of respiratory distress in new borns.

\begin{tabular}{|l|l|l|l|}
\hline & $\begin{array}{l}\text { Neonates with } \\
\text { respiratory distress }\end{array}$ & $\%$ & Significance \\
\hline Group N & 17 & 28.33 & $\mathrm{P}=0.008$ \\
\hline Group S & 5 & 8.33 & Significant \\
\hline
\end{tabular}

$17(28.33 \%)$ and $5(8.33 \%)$ neonates from group $\mathrm{N}$ and group $\mathrm{S}$ were admitted in NICU for respiratory distress. The difference was found to be statistically significant $(\mathrm{P}=0.008)$ (Table 7).

Table 7: Incidence of admissions in NICU for respiratory distress.

\begin{tabular}{|c|c|c|c|}
\hline & $\begin{array}{l}\text { Admissions } \\
\text { in NICU }\end{array}$ & $\%$ & Significance \\
\hline Group N & 17 & 28.33 & $\mathrm{P}=0.008$ \\
\hline Group S & 5 & 8.33 & Significant \\
\hline
\end{tabular}

Mechanical ventilation was required in $3(5 \%)$ neonates from group $\mathrm{N}$ whereas 1 neonate from group $\mathrm{S}$ required mechanical ventilation $(1.67 \%)$. The difference was not found to be statistically significant $(\mathrm{P}=0.61)$ (Table 8).

Table 8: Need for mechanical ventilation in neonates.

\begin{tabular}{|l|l|l|l|}
\hline & $\begin{array}{l}\text { Need for mechanical } \\
\text { ventilation }\end{array}$ & $\%$ & Significance \\
\hline Group N & 3 & 5.0 & \\
\hline Group S & 1 & 1.67 & $\mathrm{P}=0.61 *$ \\
\hline *Not significant & & \\
\hline
\end{tabular}

The average stay in NICU was $5.82 \pm 1.91$ days and $3.78 \pm 1.24$ days for group $\mathrm{N}$ and Group $\mathrm{S}$ respectively. The difference was found to be statistically significant $(\mathrm{P}<0.0001)$ (Table 9). All neonates were successfully discharged from the NICU and there was no neonatal mortality in any neonate of the studied groups.

Table 9: Mean NICU stay in neonates.

\begin{tabular}{|l|l|l|l|}
\hline & $\begin{array}{l}\text { Mean } \\
\text { NICU stay }\end{array}$ & $\begin{array}{l}\text { Std } \\
\text { deviation }\end{array}$ & Significance \\
\hline Group N & 5.82 & \pm 1.91 & $\begin{array}{l}\mathrm{P}<0.0001 \\
\text { Significant }\end{array}$ \\
\hline Group S & 3.78 & \pm 1.24 & S \\
\hline
\end{tabular}

\section{DISCUSSION}

The administration of corticosteroids to pregnant women who are at risk of preterm labor is proved to be one of the most effective interventions to prevent neonatal morbidity and mortality. It is proved to reduce the incidence of respiratory distress in the neonates and consequently reduce the need for admissions in neonatal intensive care units. The American College of Obstetricians and Gynecologists have recommended that corticosteroids be given to pregnant women between 24 weeks and 33 weeks of gestation who are at risk of preterm delivery within 7 days, including for those with ruptured membranes and multiple gestations. ${ }^{11}$ The beneficial effect of corticosteroid administration to pregnant women for prevention of neonatal respiratory distress has been confirmed by various studies conducted by authors such as Roberts D et al and Gonzales LW et al. ${ }^{12,13}$

Carlo W et al conducted a study of 4924 infants with an aim to determine if use of antenatal corticosteroids is associated with improvement in major outcomes for infants born at 22- and 23-weeks' gestation. ${ }^{14}$ The main outcome measure was mortality and neurodevelopmental impairment at 18 to 22 months' corrected age. The authors found that if the mothers had received antenatal corticosteroids, the following events occurred significantly less in infants born at 23,24 , and 25 weeks' gestation: death by 18 to 22 months, hospital death, intraventricular hemorrhage, or periventricular leukomalacia and necrotizing enterocolitis. For infants born at 22 weeks' gestation, the only outcome that occurred significantly less was death or necrotizing enterocolitis $\mathbf{7 3 . 5 \%}$ with exposure to antenatal corticosteroids vs. $84.5 \%$ without exposure; AOR, 0.54 [95\% CI, 0.30-0.97]). On the basis of these findings the authors concluded that antenatal exposure to corticosteroids compared with no exposure was associated with a lower rate of death or neurodevelopmental impairment at 18 to 22 months. Similar positive effects on neonatal morbidity and mortality were reported by the authors like Guinn DA et al and Crowther CA et al. ${ }^{15,16}$

Role of corticosteroid administration in near term or term gestation has been controversial and different studies have reported different outcomes in terms of incidence of respiratory distress in neonates, morbidity and mortality. The study conducted by Ashraf $\mathrm{N}$ et al didn't find any significant correlation between antenatal corticosteroid administration and reduction in incidence of respiratory distress in neonates and concluded that Prophylactic antenatal corticosteroid for elective cesarean delivery between 34 and 37 weeks is not effective in improving neonatal outcomes. ${ }^{17}$ Similar findings were reported by Porto A.M et al. ${ }^{18}$ Saccone et al undertook a systematic review with meta-analysis to evaluate the effectiveness of antenatal corticosteroids given at $\geq 34$ weeks' gestation. ${ }^{19}$ In this review Randomized clinical trials comparing antenatal corticosteroids with placebo or no treatment in women with a singleton pregnancy at $\geq 34$ weeks' gestation and trials on antenatal steroids in women expected to deliver late preterm (340-366 weeks) and trials given before planned cesarean delivery at term $(\geq 37$ 
weeks) were included. The authors concluded that there was a significant reduction in neonatal respiratory morbidity after antenatal administration of corticosteroid to mother even at or after 34 weeks of gestation. Similar findings were reported by Peaceman AM et al. ${ }^{20}$

\section{CONCLUSION}

Antenatal administration of corticosteroids to women undergoing elective cesarean section at or near term (between 34-37 weeks) is found to have a beneficial effect in reducing incidence of transient tachypnoea of new born, respiratory distress syndrome, NICU admissions and overall NICU stay.

Funding: No funding sources

Conflict of interest: None declared

Ethical approval: The study was approved by the Institutional Ethics Committee

\section{REFERENCES}

1. Yurdakök M. Transient tachypnea of the newborn: what is new? J Matern Fetal Neonatal Med. 2010;23(3):24-6.

2. Ma CC, Ma S. The role of surfactant in respiratory distress syndrome. Open Respir Med J. 2012;6:4453.

3. Molina JJ, Jacobo OB, Valdivia JM. Hyaline membrane disease: mortality and maternal and neonatal risk factors. Ginecol Obstet Mex.2006;74(7):354-9.

4. Boughton K, Gandy G, Gairdner D. Hyaline membrane disease. II. Lung lecithin. Arch Dis Child. 1970;45(241):311-20.

5. Ramachandrappa A, Jain L. Elective cesarean section: its impact on neonatal respiratory outcome. Clin Perinatol. 2008;35(2):373-93,

6. Mwansa-Kambafwile J, Cousens S, Hansen T, Lawn JE. Antenatal steroids in preterm labour for the prevention of neonatal deaths due to complications of preterm birth. Int J Epidemiol. 2010;39(1):i12233.

7. Mylonas I, Friese K. Indications for and Risks of Elective Cesarean Section. Dtsch Arztebl Int. 2015;112(29-30):489-95.

8. Hillemanns P, Hepp H, Rebhan H, Knitza R. Emergency cesarean section--organization and decision-delivery time. Geburtshilfe Frauenheilkd. 1996;56(8):423-30.

9. Gyamfi-Bannerman C, Thom EA, Blackwell SC, Tita AT, Reddy UM, Saade GR, et al. Antenatal Betamethasone for Women at Risk for Late Preterm Delivery. N Engl J Med. 2016;374(14):1311-20.

10. Kemp MW, Jobe AH, Usuda H, Nathanielsz PW, Li C, Kuo A, Huber HF, Clarke GD,Saito M, Newnham JP, Stock SJ. Efficacy and safety of antenatal steroids. Am J Physiol Regul Integr Comp Physiol. 2018;315(4):R825-R839.

11. ACOG Committee on Obstetric Practice. ACOG Committee Opinion No. 475: antenatal corticosteroid therapy for fetal maturation. Obstet Gynecol. 2011;117(2 Pt 1):422-4.

12. Roberts D, Brown J, Medley N, Dalziel SR. Antenatal corticosteroids for accelerating fetal lung maturation for women at risk of preterm birth. Cochrane Database Systematic Reviews. 2017(3).

13. Gonzales LW, Ballard PL, Ertsey R, Williams MC. Glucocorticoids and thyroid hormones stimulate biochemical and morphological differentiation of human fetal lung in organ culture. J Clin Endocrinol Metab 1986;62(4):678-91.

14. Carlo WA, McDonald SA, Fanaroff AA, Vohr BR, Stoll BJ, Ehrenkranz RA, et al. Association of antenatal corticosteroids with mortality and neurodevelopmental outcomes among infants born at 22 to 25 weeks' gestation. Eunice Kennedy Shriver National Institute of Child Health and Human Development Neonatal Research Network. JAMA 2011;306(21):2348-58.

15. Guinn DA, Atkinson MW, Sullivan L, Lee M, MacGregor S, Parilla BV, et al. Single vs weekly courses of antenatal corticosteroids for women at risk of preterm delivery: A randomized controlled trial. JAMA 2001;286(13):1581-7.

16. Crowther CA, Doyle LW, Haslam RR, Hiller JE, Harding JE, Robinson JS. Outcomes at 2 years of age after repeat doses of antenatal corticosteroids. ACTORDS Study Group. N Engl J Med 2007;357(12):1179-89.

17. Ashraf Nabhan, Al-Helaly A, Ramadan A. Prophylactic antenatal corticosteroid before elective cesarean delivery at or near term to improve perinatal outcome. Med J Cairo Univ. 2014;82(1).

18. Porto AM, Coutinho IC, Correia JB, Amorim MM. Effectiveness of antenatal corticosteroids in reducing respiratory disorders in late preterm infants: randomized clinical trial. BMJ. 2011;342:d1696

19. Saccone G, Berghella V. Antenatal corticosteroids for maturity of term or near term fetuses: systematic review and meta-analysis of randomized controlled trials. BMJ. 2016;355:i5044.

20. Peaceman AM, Bajaj K, Kumar P, Grobman WA. The interval between a single course of antenatal steroids and delivery and its association with neonatal outcomes. Am J Obstet Gynecol. 2005;193(3):1165-9.

Cite this article as: Sharfuddin S, Pradhan A, Imam MN. Prophylactic antenatal corticosteroid before elective cesarean delivery at or near term: a retrospective study. Int J Reprod Contracept Obstet Gynecol 2019;8:927-31. 